\title{
Augmented Virtuality for Arthroscopic Knee Surgery
}

\author{
John M. Li ${ }^{1}$, Davide D. Bardana ${ }^{2}$, and A. James Stewart ${ }^{1,2}$ \\ 1 School of Computing, Queen's University \\ ${ }^{2}$ Human Mobility Research Centre, Kingston General Hospital
}

\begin{abstract}
This paper describes a computer system to visualize the location and alignment of an arthroscope using augmented virtuality. A 3D computer model of the patient's joint (from CT) is shown, along with a model of the tracked arthroscopic probe and the projection of the camera image onto the virtual joint. A user study, using plastic bones instead of live patients, was made to determine the effectiveness of this navigated display; the study showed that the navigated display improves target localization in novice residents.
\end{abstract}

\section{Introduction}

Arthroscopic knee surgery is a minimally invasive procedure in which the surgeon navigates a surgical tool using camera images displayed on a screen above the patient. A small incision allows the arthroscope to provide a view of the surgical site while inducing less trauma than comparable open surgery.

However, navigating within the joint is challenging because the camera image on the overhead display has an unintuitive relationship with the arthroscope in the surgeon's hand, making hand/eye coordination very difficult. Surgeons must make a mental coordinate transformation to become correctly oriented within the patient.

This paper describes a navigation system to visualize the arthroscope and probe in relation to a virtual model of the patient's joint (Figure1). The navigation system also shows the camera image projected onto the virtual joint to make explicit the relationship between the camera image and the patient's anatomy.

This approach is referred to as "augmented virtuality" (AV), where a virtual view is augmented with real world camera images. In the navigation system, the $\mathrm{AV}$ view is shown beside the traditional arthroscope view.

A user study on a knee simulator was done to determine whether the system improves performance in a task of locating particular areas within a knee joint. We hypothesized that the system would improve the performance of novice residents, while doing little to help or hinder the performance of experienced surgeons.

Subjects from novice surgical residents to highly skilled surgeons performed tasks on a knee simulator with and without the aid of the navigation system. Correctness, task completion time, and tool path length were recorded and compared to determine the effectiveness of the system.

G. Fichtinger, A. Martel, and T. Peters (Eds.): MICCAI 2011, Part I, LNCS 6891, pp. 186-193, 2011.

(C) Springer-Verlag Berlin Heidelberg 2011 


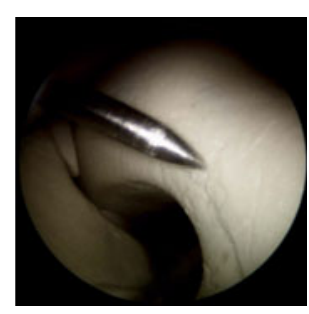

(a)

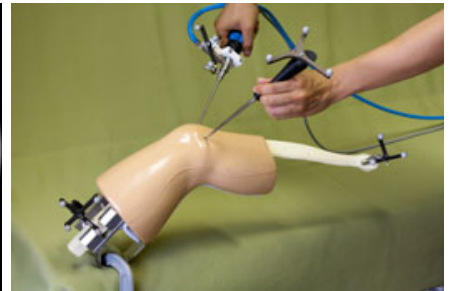

(b)

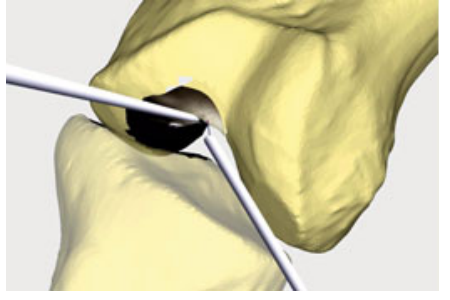

(c)

Fig. 1. (a) The standard arthroscopic image, which gives few clues to the location of the camera and probe within the joint. (b) The system setup with optical trackers attached to the camera, probe, femur, and tibia. (c) The navigated view showing camera, probe, femur, and tibia. The camera image is projected onto the bones and is visible as the darker area on the femoral condyle.

\section{Related Work}

In the context of arthroscopic surgery, a number of authors have presented surgical navigation systems that show relative tool positions. But, to the best of our knowledge, none augment the virtual model with live camera images.

Tonet et al. 1] described an augmented reality navigation system for knee surgery that shows a 3D model of the patient's anatomy along with the relative tool positions. The field of view of the arthroscope was also dynamically highlighted on the 3D model, but no camera image was shown. No formal user study was made to evaluate the system.

Monahan et al. 2] presented a navigation system for arthroscopic hip surgery that tracks tool positions with linkage encoders and displays their relative positions in various $3 \mathrm{D}$ views. They performed a user study to determine if their navigation system could help increase speed and accuracy. They found a reduction in the length of the path travelled by the tool and in completion time when using their system.

A number of arthroscopic training systems that include virtual views have also been proposed. Heng et al. 3] used virtual models of human anatomy to devise a virtual reality training simulation for arthroscopic surgery. They developed a haptics device to simulate force feedback within a "black box" to present the user with a purely virtual environment. Along with force feedback, their system showed simulated and external views of the arthroscope to aid in training. A user study was not performed to validate their system as a training tool. In a similar way, Bayona et al. 4] developed a shoulder arthroscopy training simulator with force feedback. Their interface included a virtual external rendering which showed the viewing cone of the arthroscope, but without live images. They performed user studies to validate the device as a training tool and showed that it was more useful for inexperienced surgeons than for experienced surgeons. 
Although augmented virtuality has not been widely used in arthroscopic procedures, a number of authors have developed AV systems for navigation in various other endoscopic procedures.

Paul et al. 5] compared AR and AV approaches using a computer system that mixed images from a surgical microscope with 3D preoperative images. They found that the augmented virtuality approach complemented the augmented reality approach because it provided a better understanding of the spatial relationship between the surgical site and the $3 \mathrm{D}$ preoperative images.

Dey et al. [6] explored the concept of mixed reality to map intra-operative endoscopic video to a patient's anatomy. They fused images of a brain phantom from a tracked endoscope to surfaces derived from 3D preoperative images. Their focus was to paint the video images onto the 3D surface and impart stereoscopic depth cues to provide assistance in surgical planning and guidance. Liao et al. [7] developed a system that fused endoscopic image mosaics with a 3D ultrasound image model to provide extended visualization in intrauterine fetal surgery. Nicolau et al. 8] presented a guidance system for laparoscopic surgery which showed both AR and AV views.

The contributions of this paper are (a) an arthroscopic navigation system that projects the camera view onto the joint and (b) a user study showing that the system can improve performance of target localization in novice residents.

\section{System Description}

The arthroscope hardware consisted of an IM4000/IM4120 high definition camera system and an HD4300 4mm 30 degree arthroscope from Conmed Linvatec. The knee was modelled with a "Sawbones" artificial knee joint \#1413 from Pacific Research Laboratories. The proximal end of the femur was clamped to a table, allowing the tibia to be moved relative to the femur, as is commonly done in surgery.

The components of the system were tracked with a Polaris Hybrid optical tracking system from Northern Digital. Four passive trackers from Traxtal were attached to the arthroscope, the probe, the femur, and the tibia. For the arthroscope and probe, custom mounts were designed and built to hold the trackers. For the femur and tibia, the trackers were held in place by a standard percutaneous clamp.

A thirty inch monitor was positioned in portrait orientation with its bottom edge two meters above the floor, about two meters beyond the knee as seen by the surgeon. (This is a common position for the monitor during arthroscopic surgery.) The standard arthroscopic view was shown on the lower half of the screen. The AV navigation display was in the upper half, showing a 3D computer model of the joint, along with models of the arthroscope and the probe, and the projection of the live arthroscopic image onto the joint (Figure $2 \mathrm{a}$ ). The AV view was rendered from the same viewpoint, relative to the virtual bones, as the subject saw the plastic bones.

Fiducial markers were fixed to the femur and tibia and a CT scan of those bones was made. Mesh models of the bones were made from the CT scan and the 


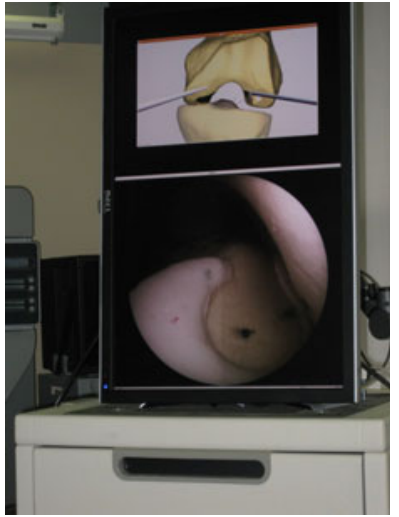

(a)

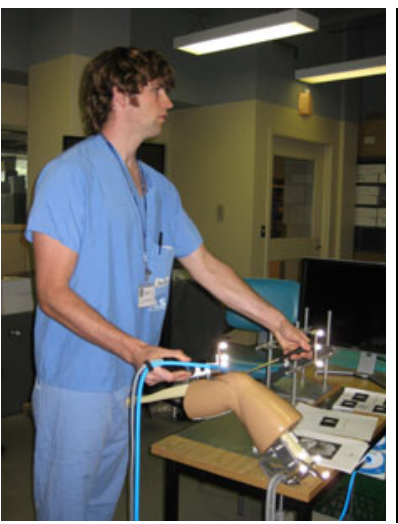

(b)

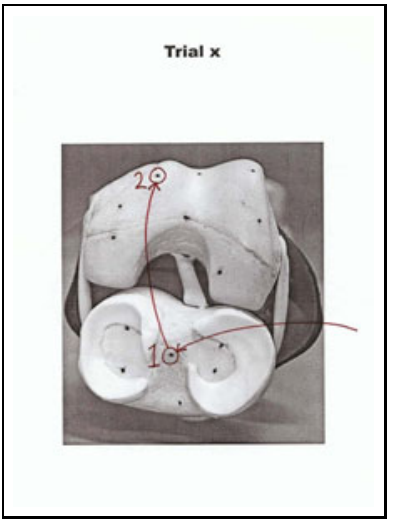

(c)

Fig. 2. (a) The monitor showing (top) the navigated view and (bottom) the standard arthroscopic image. (b) One of the residents performing a trial. (c) The instruction sheet given for one trial indicating the target points. There are eight target points on the femoral condyles and intracondylar notch (top) and six target points on the tibial plateau (bottom).

locations of the fiducials in the CT coordinate space were recorded. A pairedpoint algorithm used the fiducial locations to establish the bone-to-tracker transformation. The fiducials were not subsequently used as targets in the target localization task.

In order to project the arthroscopic image onto the bone, the OpenCV Computer Vision Library was used to find the intrinsic and extrinsic calibration parameters of the arthroscope camera. OpenGL was used to render a model of the arthroscope, along with the arthroscopic image projected (using GL_REPLACE) onto the virtual bone from the camera position.

The system was limited in that the camera head could not be rotated about its axis, as can be done during surgery, because we did not have the necessary equipment to track the camera head.

\section{Experiment Methodology}

Eight orthopaedic surgeons and fourteen orthopaedic residents with varying degrees of arthroscopic experience were tested. Each subject performed twelve trials (Figure 2b). In each trial, the subject was asked to locate two target points within the knee joint. The subject would locate the first target point in the arthroscope image, then locate the second target point in the arthroscope image. The sub-millimeter accuracy of the Polaris Hybrid was sufficient for this task. In a variant of the trial, the subject would also touch the probe to each target point. 
The trials were performed in four blocks. Each block consisted of three of the same type of trial, but with three different pairs of points. The block order was randomized, as was the trial order within each block. The trials in the four blocks were:

1. Using only the arthroscope image, center each point in the camera view.

2. Using both the arthroscope image and the navigation display, center each point in the camera view.

3. Using only the arthroscope image, center each point in the camera view, then touch it with the probe.

4. Using both the arthroscope image and the navigation display, center each point in the camera view, then touch it with the probe.

The same three pairs of points were used in block 1 and block 2 to allow paired comparison of the results with and without the navigation display. A different set of three pairs was used for both block 3 and block 4 . Blocks 1 and 2 were always separated by a different block, as were blocks 3 and 4 . In the trials that used only the arthroscope image, the navigation display was turned off.

There were eight target points on the femur and six on the tibia. The locations of the targets were chosen according to a surgeon's description of commonly scoped locations on the femur and tibia. The target points were marked with a black marker on the bone (Figure $2 \mathrm{c}$ ).

For each trial, the subject was shown a picture of the exposed femur and tibia, with the two target points circled and arrows indicating the order in which they were to be found (Figure 25). Every trial included one target from the femur and one target from the tibia.

Each subject was shown the layout of the targets on a knee model without the skin covering. The trials were described, and the subject was given ten minutes using the arthroscope and probe to familiarize themselves with the locations of the different targets inside the knee joint. For each trial, we measured the following:

1. Time to locate the target in the camera view.

2. Distance travelled by the camera tip before locating the target.

3. Whether the correct target was found.

4. Time to position the probe on the target (blocks 3 and 4 only).

5. Distance travelled by the probe tip before being positioned on the target (blocks 3 and 4 only).

For the first target, we measured the time and distance from the entry portal on the skin surface until the subject declared that the target was found (even if the wrong target was found). For the second target, we measured the additional time and distance from the first target until the subject declared that the second target was found.

The subject started each trial with the tools in hand and outside of the skin surface. When the subject found a target point, he or she would verbally notify us and we would record the time in the log file, as well as a snapshot of the 
Table 1. Experimental results. The bold $p$ values are statistically significant. "NAV" denotes the navigated display, while "STD" denotes the standard display.

\begin{tabular}{|l|ccc|ccc|ccc|}
\hline Measurement & \multicolumn{3}{|c|}{ residents $<2$ years } & \multicolumn{3}{c|}{ residents $\geq 2$ years } & \multicolumn{3}{c|}{ surgeons } \\
& \multicolumn{3}{|c}{$\mathrm{N}=8$} & \multicolumn{3}{c|}{$\mathrm{N}=6$} & \multicolumn{3}{c|}{ N =8 } \\
& NAV & STD & p & NAV & STD & p & NAV & STD & p \\
\hline correctness (\%) & 91.3 & 79.6 & $\mathbf{. 0 0 2}$ & 90.1 & 86.1 & .130 & 90.4 & 86.9 & .265 \\
camera time (sec) & 24.3 & 36.6 & $\mathbf{. 0 3 5}$ & 26.7 & 18.9 & $\mathbf{. 0 0 1}$ & 18.1 & 17.0 & .262 \\
camera distance (cm) & 14.0 & 18.1 & $\mathbf{. 0 7 2}$ & 14.3 & 12.1 & $\mathbf{. 0 3 8}$ & 11.6 & 11.8 & .439 \\
probe time (sec) & 20.2 & 24.8 & .182 & 34.1 & 23.0 & $\mathbf{. 0 1 7}$ & 17.1 & 19.4 & .260 \\
probe distance (cm) & 13.8 & 19.5 & $\mathbf{. 0 4 1}$ & 18.6 & 14.5 & .079 & 10.8 & 14.1 & .094 \\
\hline
\end{tabular}

arthroscopic image for later verification. The log also continuously recorded the position of both the arthroscope tip and the probe tip, so that we could later calculate the distances travelled. A questionnaire was administered after the trial.

\section{Results}

The measured experimental results are shown in Table1. Fifteen hypotheses were tested: For each of the three subject groups and each of the five performance measurements, we performed a t-test to determine whether the performance measurement using the navigated display was superior to that measurement using only the standard display.

For residents with less than two years of experience, there was a significant improvement in acquiring the correct target $(91.3 \%$ versus $79.6 \%, \mathrm{p}=0.002)$, in the time taken to locate the target with the camera (24.3 seconds versus 36.6 seconds, $\mathrm{p}=0.035)$, and in the distance travelled by the probe to touch the target $(13.8 \mathrm{~cm}$ versus $19.5 \mathrm{~cm}, \mathrm{p}=0.041)$.

For residents with at least two years of experience, there was a significant worsening in the time to locate the target with the camera (26.7 seconds versus 18.9 seconds, $\mathrm{p}=0.001$ ), in the distance travelled by the camera to locate the target $(14.3 \mathrm{~cm}$ versus $12.1 \mathrm{~cm}, \mathrm{p}=0.038)$, and in the time for the probe to touch the target (34.1 seconds versus 23.0 seconds, $\mathrm{p}=0.017$ ).

For surgeons, no significant differences were found between the navigated and standard methods.

From the questionnaires, which gathered responses on a seven-point Likert scale:

- Subjects at all three levels agreed that the navigated display gave a better understanding of the 3D configuration of the bone, tool, and camera.

- Subjects were neutral or agreed that it was easier to find and touch the fiducials using the navigated display instead of only the traditional arthroscopic image. 
- Novice residents and surgeons were neutral that the lack of camera rotation made the task more difficult, while experienced residents strongly agreed.

- Surgeons agreed that the experimental setup accurately simulated what they would experience in the operating room, while residents, as a group, were neutral.

\section{Discussion}

The navigated display is clearly beneficial to residents with less than two years of arthroscopic experience. This strongly supports our hypothesis that the navigated display can assist early residents.

The navigated display is clearly detrimental to residents of at least two years of arthroscopic experience. We believe that this is because those residents have already established a mental model of the arthroscopic environment which is different than the $3 \mathrm{D}$ model that the $\mathrm{AV}$ navigation system provided. In fact, one experienced resident explicitly said that his mental model clashed with that of the navigated display.

We could not find any significant effect of the navigated display upon surgeons, all of whom had at least five years of arthroscopic experience as surgeons. We believe that this is because the surgeons were so highly trained that they did not need, and hence paid little attention to, the navigated display. However, five of the eight surgeons noted that they occasionally used the display to confirm the location of target points that were harder to find, so there is potential for intraoperative use by surgeons. In addition, six out of eight surgeons commented that the system would be beneficial as a training tool for novice residents.

The experimental setup seems to be close to what would be experienced in surgery, according to the experienced surgeons. But the lack of camera rotation made the task more difficult than would be encountered in real surgery.

All subjects believed that the navigated display gave a better understanding of the 3D configuration of the bone, tool, and camera. Although no group of subjects strongly believed that the navigated display made the target points easier to locate, we measured a substantial performance improvement among the novice residents. In our observations and interviews of the novice and experienced residents, we found that they used the navigated display to locate the general area of the target, then used the traditional arthroscopic view to adjust their final position. That suggests that the live camera view projected onto the bone may not be necessary.

\section{Conclusion}

The study shows strong evidence that the AV navigated display can assist novice residents in the difficult task of locating anatomical locations in arthroscopic surgery.

The contrary results from experienced residents suggest that many of those residents form a mental model of the procedure that is different from our $3 \mathrm{D}$ 
model. Further study should be done to determine this trained mental model. Either our 3D model could be adapted to the trained model, or another study could be performed to compare the utility of the trained model to that of our 3D model.

The most important improvement in the navigated system would be to incorporate camera rotation, although the surgeons of our study agreed that our system accurately reflected what would be experienced in the operating room.

Acknowledgements. Thank you to our subjects for their participation and to the reviewers for their helpful comments. This work is supported by Natural Sciences and Engineering Research Council of Canada (NSERC) grant STPGP 336797 and by the joint NSERC/Canadian Institutes of Health Research CHRP grant 385959 .

\section{References}

1. Tonet, O., Megali, G., D'Attanasio, S., Dario, P., Carrozza, M.C., Marcacci, M., Martelli, S., La Palombara, P.F.: An augmented reality navigation system for computer assisted arthroscopic surgery of the knee. In: Delp, S.L., DiGoia, A.M., Jaramaz, B. (eds.) MICCAI 2000. LNCS, vol. 1935, pp. 1158-1162. Springer, Heidelberg (2000)

2. Monahan, E., Shimada, K.: A study of user performance employing a computeraided navigation system for arthroscopic hip surgery. International Journal of Computer Assisted Radiology and Surgery 2(3-4), 245-252 (2007)

3. Heng, P.A., Cheng, C.Y., Wong, T.T., Xu, Y., Chui, Y.P., Chan, K.M., Tso, S.K.: A virtual-reality training system for knee arthroscopic surgery. IEEE Transactions on Information Technology in Biomedicine 8(2), 217-227 (2004)

4. Bayona, S., Fernández-Arroyo, J.M., Martín, I., Bayona, P.: Assessment study of insight ARTHRO VR arthroscopy virtual training simulator: face, content, and construct validities. Journal of Robotic Surgery 2(3), 151-158 (2008)

5. Paul, P., Fleig, O., Jannin, P.: Augmented virtuality based on stereoscopic reconstruction in multimodal image-guided neurosurgery: methods and performance evaluation. IEEE Transactions on Medical Imaging 24(11), 1500-1511 (2005)

6. Dey, D., Gobbi, D.G., Slomka, P.J., Surry, K.J.M., Peters, T.M.: Automatic fusion of freehand endoscopic brain images to three-dimensional surfaces: creating stereoscopic panoramas. IEEE Transactions on Medical Imaging 21(1), 23-30 (2002)

7. Liao, H., Tsuzuki, M., Mochizuki, T., Kobayashi, E., Chiba, T., Sakuma, I.: Fast image mapping of endoscopic image mosaics with three-dimensional ultrasound image for intrauterine fetal surgery. Minimally Invasive Therapy \& Allied Technologies 18(6), 332-340 (2009)

8. Nicolau, S.A., Goffin, L., Soler, L.: A Low Cost and Accurate Guidance System for Laparoscopic Surgery: Validation on an Abdominal Phantom. In: Proceedings of the ACM Symposium on Virtual Reality Software and Technology, pp. 124-133. ACM, New York (2005) 